\title{
Modelling the micro- and macro-structure efficiencies of a compliant petiole beam
}

\author{
D. Pasini \\ Mechanical Engineering, McGill University, Montreal, Canada
}

\begin{abstract}
Structural geometry and material properties govern the way in which a petiole complies to wind and gravity forces. Earlier works that examined the mechanical efficiency of a petiole undergoing twisting and bending proposed simplified models for its geometry and material. The former was approximated with a theoretical semi-elliptical contour, while the latter was viewed as a homogeneous material. These simplifications are refined in this paper, which presents progress on both issues. The petiole is considered as a hierarchical structure with differently sized structural features. A multiscale model of the twist to bend ratio is presented to capture the structural efficiencies of the petiole at both the macroand micro-scale. Dimensionless factors and shape parameters are introduced to describe the coupling effect of the efficiency gained at different levels of the length scale. The results are plotted into efficiency maps showing how structural hierarchies impact the compliant properties of a petiole beam.
\end{abstract}

Keywords: microstructure, structural hierarchy, shape, twist to bend ratio.

\section{Introduction}

A leaf petiole can be considered as a hierarchical solid that contains structural features at different length scales. Cellulose is the material building block, which is shaped during growth into several structural hierarchies. At each hierarchical level, the elements repeat themselves to form ordered patterns. The architecture of these patterns and the way in which they are nested into each other plays a crucial role in the overall mechanical responses of the petiole.

Flexibility is a property each plant possesses to varying degrees and specific to each species. As other motile and sessile organisms, leaf petioles exhibit the ability to adjust their posture in response to applied external forces. Flexibility is 
beneficial to a petiole, since it allows the reduction of flow-induced drag of the wind, thereby decreasing the need for structural resistance, material use, and energy.

Compliance of biological beams has been a subject of interest for many biologists in the last two decades [1-8]. The compliance to torsion, as opposed to bending, was the focus of experimental and theoretical investigations, based on continuum mechanics. The material was assumed to be continuous and homogenous, and the cross-section geometry was limited mainly to circular shapes. More recent works examined the impact alternative geometries may have on the twist to bend ratio [7-8]. Ideal superellitpical cross-sections, including triangular top grooved shapes, were studied. In these works, however, the contribution of material microstructure to the petiole resistance was ignored.

In this paper, a multiscale model based on continuum mechanics is proposed to capture the influence cross-section geometry and material anisotropy have on the twist to bend ratio of petiole beams. In section 3, a general model is introduced to characterize hierarchical structures and applied to leaf petioles in section 4. Compliance properties of theoretical natural structured shapes are plotted on maps for comparison of several petiole specimens. The maps show that structuring cellulose at different hierarchical levels, from the tissue to the cross-section, impacts the petiole resistance to external forces.

\section{Hierarchies in the petiole structure}

Like other living organisms, the design of a leaf petiole results to meet multifunctional and evolving requirements. Beyond accomplishing vital functions, most organisms structure their bodies to provide skeleton support. Despite the diversity of material use, e.g. calcium carbonate, silica, chitin and cellulose, most organisms share a common feature. Their material has a complex structural organization, which reveals a particular design at each length scale. Various levels of structural hierarchies can be nested in natural organisms, such as those present in trabecular bones, wood, and tendon. For example, in the Euplectella aspergillum, a deepwater sponge, six hierarchical levels have been recently identified [9]. Their levels of structuring range from the nano- to the macroscopic scale. Each one has its own structural design at a particular order, and their formation occurs at different growth phases. In this case, the assembly consists of a three-dimensional cylindrical lattice structure, where the interaction among structural motifs of each order plays a crucial role. Material structuring confers remarkable physical properties, such as strength, toughness, and resilience, with minimum material use.

In a leaf petiole, the material is fibrous and is also assembled hierarchically. In general, there are three tissues, which make up the dermal, vascular and ground systems. Each tissue has distinct characteristics, with patterns that may vary among species, as shown in Figure 1. In the case of dicots, the material consists of tracheids, which are tubular cells in the xylem of vascular plants. Their function is not limited to structural support, since each cell has a vacuole filled with nutrient liquid. From a structural point of view, however, the 
contribution of the sap is negligible and the cells may be assumed to be hollow cellulose tubes. At the nano-scale, each cellular tube is embedded in a hemicellulose-lignin matrix reinforced by fibrils of about $2.5 \mathrm{~nm}$ diameter, wound helically at specific angles around the cell (Fig. 2(a)).
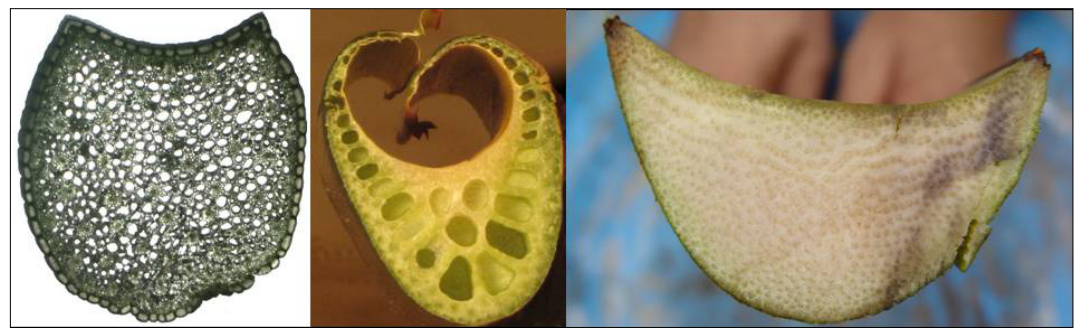

Figure 1: Petiole micro-structures. Species from left: (a) Monstera, (b) Musaceae, (c) Palm.

Although the largest size structure of the hierarchy often plays the most relevant role, each hierarchy contributes to the overall mechanical performance. The next section proposes a scheme to characterize the effect of material hierarchies.
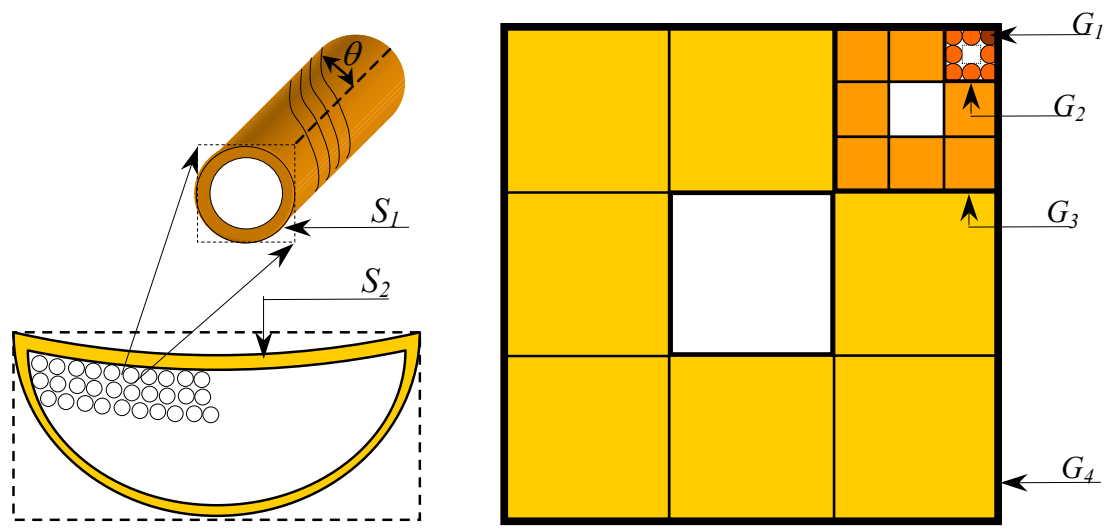

Figure 2: (a) Structural hierarchies of a petiole. (b) Structuring of an ideal cross-section; it applies to all cell components, though it is shown for one cell at each order only.

\section{A model for structural hierarchies}

Application of theory of elasticity is not restricted to a specific length scale. Classic mechanics can predict the deformation of a structural configuration at any length scale. For a multi-scale structure, such as the petiole, the theory can provide accurate results as long as the structure size at one level is significantly different from those that proceed or follow in the hierarchy. 
The hierarchical order of a structure may be defined as the number $n$ of scale levels with recognizable structure [10-12]. To describe the structuring effect of a material, we examine an idealized example. Figure 2(b) shows a cross-section structured in four hierarchies, where the elements are assumed to be continuous at each level. At level 0 , the material $M_{0}$ is uniform and shapeless. When shaped, $M_{0}$ becomes a solid circular two dimensional structure. During this process, $M_{0}$ is conferred with geometrical properties $G_{l}$ and $M_{l}$, i.e. the shaped material at level $\mathrm{n}=1$, inherit properties $M_{0} G_{1}$. As an example, we apply the scheme of shape transformers, $\psi_{G}$, to the bending stiffness [13,14]. The effective flexural properties $E_{l}$ at the first hierarchical order are obtained by normalizing $E_{0} I_{l}$ with the envelope property $I_{D I}$ :

$$
E_{1}=E_{0} \frac{I_{1}}{I_{D 1}}=E_{0} \psi_{I}^{1}
$$

At the following level, the circular units are clustered together to form a hollow square cross-section. The effective properties of $M_{l}$, e.g. $E_{l}$, can be considered uniform and isotropic; as a result, structuring at level $\mathrm{n}=2$ confers properties $G_{2}$ to material $M_{l}$ (Fig. 2(a)). The effective flexural modulus can be expressed as:

$$
E_{2}=E_{1} \frac{I_{2}}{I_{D 2}}=E_{1} \psi_{I}^{2}=E_{0} \psi_{I}^{1} \psi_{I}^{2}
$$

Here, the structuring of the two hierarchies is factored in by the shape properties, whose factorial determines the effective material properties. In turn, further structuring of the cells in the second level results in a hollow square crosssection with geometric properties $G_{3}$. Thus, the effective Young's modulus at level 3 is transformed to $E_{3}=E_{2} \psi_{I}^{3}=E_{0} \psi_{I}^{1} \psi_{I}^{2} \psi_{I}^{3}$. By extrapolation, structuring a material $M_{0}$ in $n$ hierarchical levels can be described by:

$$
\frac{E_{n}}{E_{0}}=\prod_{j=1}^{n} \psi_{I}^{j}
$$

The above relation is a ratio of effective material properties. In order to factor in the overall structure size, $E_{n}$ in equation (3) can be rearranged and substituted in the shape transformers expression of an Equation of Mechanics, E.M. [13]. Mass, torsional, and flexural stiffness can be written as:

$$
\text { E.M. }=F \times M_{0} \times \prod_{j=1}^{n} S^{j} \times G_{D}
$$

where $F$ are problem constants, $G_{D}$ the geometric quantity of the overall envelope, and $S$ describes the shape properties at each level of the hierarchy.

\section{Multi-scale model for a layered micro-structured petiole}

In Figures 1 and 2(a), two layers can be identified in the petiole cross-section, each with its own material properties. The inner layer is a structured material of uniformly distributed hollow tubular cells. The outer is the dermal tissue, which consists of peripheral bands of lignified material. The properties of the former 
are described by relation (3). The contribution of the latter depends on the thickness of the outer coat, which is often species-specific. The materials properties resulting from layering a system with $k$ materials can be expressed in terms of shape transformers [14]. For example, the effective flexural modulus of a multilayered system, where layering a material $M_{i}$ is described by $\psi_{l i}$, is given

$$
E_{T}=\sum_{i=1}^{k} E_{i} \psi_{I i}
$$

If among the layers, there are structured materials, then their contribution can be factored in by replacing $E_{n}$ in eq. 3 , with $E_{i}$ in eq. (5), namely:

$$
E_{T}=\sum_{i=1}^{k}\left(E_{o} \prod_{j=1}^{n} \psi_{I}^{j}\right) \psi_{I i}
$$

Then, Equation (4) can be generalized to include the effect of materials layering:

$$
E . M .=F \times M_{o} \times \sum_{i=1}^{k}\left(\prod_{j=1}^{n} S^{j}\right) S_{i} \times G_{D}
$$

The above rationale can be applied to assess the compliance of a petiole cantilever under wind and gravity forces. Therefore, the bending to twist ratio of a petiole with prescribed length boundary conditions can be written as:

$$
\frac{E I}{G J_{t}}=\sum_{i=1}^{k} \frac{\left(E_{0} \prod_{j=1}^{n} \psi_{I}^{j}\right) \psi_{I i}}{\left(G_{0} \prod_{j=1}^{n} \psi_{J}^{j}\right) \psi_{J i}} \frac{I_{D n}}{J_{D n}}=2(1+v) \sum_{i=1}^{k} \frac{\left(\prod_{j=1}^{n} \psi_{I}^{j}\right) \psi_{I i}}{\left(\prod_{j=1}^{n} \psi_{J}^{j}\right) \psi_{J i}} \frac{I_{D n}}{J_{D n}}
$$

where $G$ is the shear modulus, $J_{t}$ the torsional constant and $v$ is the Poisson ratio, which is approximately 0.25 for plant cellulose.

Shape transformers in relation (7) were previously formulated for idealized pure geometric shapes. Here, we refine their formulation by using the Gielis parameterization of the Lame' curves [15]. This enables the accurate representation of natural forms and is used here to capture the last hierarchy of the petiole, i.e. the hollow grooved contour. In polar coordinates $(r, \phi)$, the shape contour is given by:

$$
r=f(\phi) \frac{1}{\sqrt[n_{1}]{\left(\left|\frac{1}{a} \cos \left(\frac{4}{m} \phi\right)\right|\right)^{n_{2}}+\left(\left|\frac{1}{b} \cos \left(\frac{4}{m} \phi\right)\right|\right)^{n_{3}}}}
$$

The parameters $a, b, m, n_{1}, n_{2}$ given in Table 1 , were used to plot shape contours of alternative petioles. Their respective shape transformers were formulated by resorting to parallel programming to reduce time for integral computations. 


\section{Efficiency maps}

In this section, expression (7) is plotted for the properties of the Gielis parameterized petioles (Table 1) and compared to those of 18 petiole specimens of the Monstera and Rhabarbarum species (Table 2). The flexural stiffness and the twist to bend ratio are examined for both a uniform (Figures 3-4) and a structured (Figures 5-6) material with different cell wall thicknesses.

Table 1: Gielis parameterization of petiole cross-section of different species.

\begin{tabular}{|c|c|c|c|c|c|c|c|c|c|}
\hline Petiole & Graphical & Obtained & $\left.\alpha 9^{\circ}\right)$ & \multicolumn{6}{|c|}{ Gielis Parameters } \\
\hline Specimen & sketches & contour plots & & $\mathrm{a}$ & $\mathrm{b}$ & $\mathrm{m}$ & $\mathrm{n}_{1}$ & $\mathrm{n}_{2}$ & $\mathrm{~N}_{3}$ \\
\hline $\begin{array}{l}\text { Sweetgum } \\
\text { Petiole }\end{array}$ & & & 42.8 & $\begin{array}{r}0.9 \\
f\end{array}$ & a) & $\begin{array}{l}8.3 \\
\ln (\phi)\end{array}$ & 3 & $\begin{array}{c}4 \\
\langle\phi<2\end{array}$ & $\begin{array}{l}2 \\
)\end{array}$ \\
\hline $\begin{array}{l}\text { Bean } \\
\text { Petiole }\end{array}$ & & & 45.3 & $\begin{array}{r}0.9 \\
f\end{array}$ & $\begin{array}{l}1.1 \\
\phi)=\end{array}$ & $\begin{array}{c}8 \\
\operatorname{in}(\phi)\end{array}$ & 5 & $\begin{array}{l}15 \\
\phi<2\end{array}$ & $\begin{array}{l}2 \\
)\end{array}$ \\
\hline $\begin{array}{l}\text { Monstera } \\
\text { Deliciosa }\end{array}$ & & & 42.3 & 1. & $\begin{array}{l}1.6 \\
\phi)=\end{array}$ & $\begin{array}{c}8 \\
\operatorname{in}(\phi)\end{array}$ & 70 & $\begin{array}{l}45 \\
\phi<2\end{array}$ & 25 \\
\hline $\begin{array}{l}\text { Banana } \\
\text { stem }\end{array}$ & & & 47 & 1 & $\begin{array}{c}2 \\
\phi)=\end{array}$ & $\begin{array}{c}8 \\
\operatorname{in}(\phi)\end{array}$ & 50 & $\begin{array}{l}100 \\
\phi<2\end{array}$ & $\begin{array}{l}15 \\
)\end{array}$ \\
\hline
\end{tabular}

\subsection{Uniform homogeneous material}

Figure 3 illustrates the flexural stiffness curves of ideal and natural cross-section shapes. For the former, elliptical and semi-elliptical shapes, with and without top grooves, are depicted for an increasing thickness of the outer layer from zero up to the point where the shape becomes solid. For the latter, the Gielis parameterization for natural forms (Table 2) have been plotted for petioles of Banana, Bean, Sweetgum, and Monstera species. If the elliptical shape petiole is considered as reference, then it can be seen that an increasingly sharper groove, from Sweetgum to Banana, reduces the flexural stiffness of the cross-section.

In Figure 4, points located left of the quadrant bisector twist more easily than bend for a given load. Whereas the shape transformers for flexural stiffness are size independent, for torsion this it is not the case. The curves for ideal elliptical and semi-elliptical shapes show that the flatter the shape is, the easier the crosssection is to twist. Thus, shape flattening has the effect of increasing the twist to bend ratio. It is noted that the curves for Gielis parameterized shape are plotted for the mean angles of the species, as shown in Table 1. The curves of Monstera and Sweetgum capture specimen properties of Monstera and Rhubarb species. 
Table 2: Specimen properties (1 to 12 Monstera species, 13 to $19 \mathrm{R}$. Rhabarbarum species).

Uniform Solid Shape

Structured Material

\begin{tabular}{|c|c|c|c|c|c|c|c|c|}
\hline \multirow{2}{*}{\multicolumn{2}{|c|}{ Cross-section }} & $\mathrm{B}(\mathrm{mm})$ & \multirow{2}{*}{$\lambda_{I}$} & \multirow{2}{*}{$\lambda_{J}$} & \multirow{2}{*}{$\frac{\psi_{I}}{\psi_{J}}$} & \multirow{2}{*}{$\lambda_{I}$} & \multirow{2}{*}{$\lambda_{J}$} & \multirow{2}{*}{$\frac{\psi_{I}}{\psi_{J}}$} \\
\hline & & $\mathrm{H}(\mathrm{mm})$ & & & & & & \\
\hline \multirow{2}{*}{1} & & 7.1 & \multirow{2}{*}{0.75} & \multirow{2}{*}{0.80} & \multirow{2}{*}{0.94} & \multirow{2}{*}{0.77} & \multirow{2}{*}{0.82} & \multirow{2}{*}{0.94} \\
\hline & & 7.3 & & & & & & \\
\hline \multirow{2}{*}{2} & & 6.1 & \multirow{2}{*}{0.74} & \multirow{2}{*}{0.77} & \multirow{2}{*}{0.97} & \multirow{2}{*}{0.76} & \multirow{2}{*}{0.78} & \multirow{2}{*}{0.97} \\
\hline & & 6.0 & & & & & & \\
\hline \multirow{2}{*}{3} & & 5.9 & \multirow{2}{*}{0.71} & \multirow{2}{*}{0.76} & \multirow{2}{*}{0.93} & \multirow{2}{*}{0.72} & 078 & 003 \\
\hline & & 5.8 & & & & & & \\
\hline 4 & & 5.5 & 077 & 070 & 094 & 076 & 081 & 094 \\
\hline & & 5.2 & & & & & & \\
\hline 5 & & 6.0 & 071 & 077 & 093 & 073 & 078 & 093 \\
\hline & & 5.7 & & & & & & \\
\hline 6 & & 5.5 & $r_{0}$ & 77 & 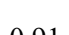 & ( 78 & 7 7 & 000 \\
\hline 0 & & 5.3 & 0.09 & 0.10 & 0.91 & 0.10 & 0.11 & 0.91 \\
\hline 7 & & 6.8 & 077 & 075 & 000 & ( 72 & 077 & 005 \\
\hline & & 7.4 & 0.11 & 0.15 & & & & \\
\hline 8 & & 6.8 & 077 & 075 & 095 & 073 & 077 & 095 \\
\hline & & 7.5 & & & & & & \\
\hline 9 & & 6.3 & 071 & 074 & 0.96 & 072 & 075 & 0.96 \\
\hline & & 6.8 & & & & & & \\
\hline 10 & & 7.1 & 07 & 076 & 003 & ( 72 & 077 & 003 \\
\hline 10 & & 7.3 & 0.11 & 0.16 & 0.93 & 0.12 & 0.11 & 0.93 \\
\hline 11 & & 6.4 & 068 & 077 & 006 & 07 & ( 72 & 006 \\
\hline 11 & & 6.9 & 0.08 & 0.11 & 0.90 & 0.10 & 0.12 & 0.90 \\
\hline 12 & & 8.4 & 072 & 077 & 005 & 074 & 078 & 005 \\
\hline 12 & & 8.5 & 0.13 & 0.17 & 0.95 & 0.14 & 0.18 & 0.95 \\
\hline 13 & & 27.0 & 075 & 073 & $10 ?$ & 076 & 074 & $10 ?$ \\
\hline 13 & & 16.0 & 0.15 & 0.13 & 1.02 & 0.16 & 0.14 & 1.02 \\
\hline 14 & & 26.0 & 073 & 077 & 094 & 074 & 078 & 094 \\
\hline & & 19.0 & & & & & & \\
\hline 15 & & 26.0 & 070 & 077 & 100 & 08 & 070 & 100 \\
\hline 15 & & 15.0 & 0.19 & 0.17 & 1.02 & 0.80 & 0.19 & 1.02 \\
\hline 16 & & 26.0 & 0.76 & 0.78 & 0.97 & 0.77 & 0.80 & 0.97 \\
\hline & & 18.0 & & & & & & \\
\hline 17 & & 21.0 & 078 & 084 & 0.93 & 07 & 085 & 0.93 \\
\hline & & 16.0 & & & & & & \\
\hline 10 & & 24.0 & ค & 7 70 & 100 & 000 & ( 70 & 100 \\
\hline 10 & & 17.0 & 0.81 & 0.18 & 1.03 & 0.82 & 0.19 & 1.03 \\
\hline
\end{tabular}

WIT Transactions on Ecology and the Environment, Vol 114, (C) 2008 WIT Press www.witpress.com, ISSN 1743-3541 (on-line) 


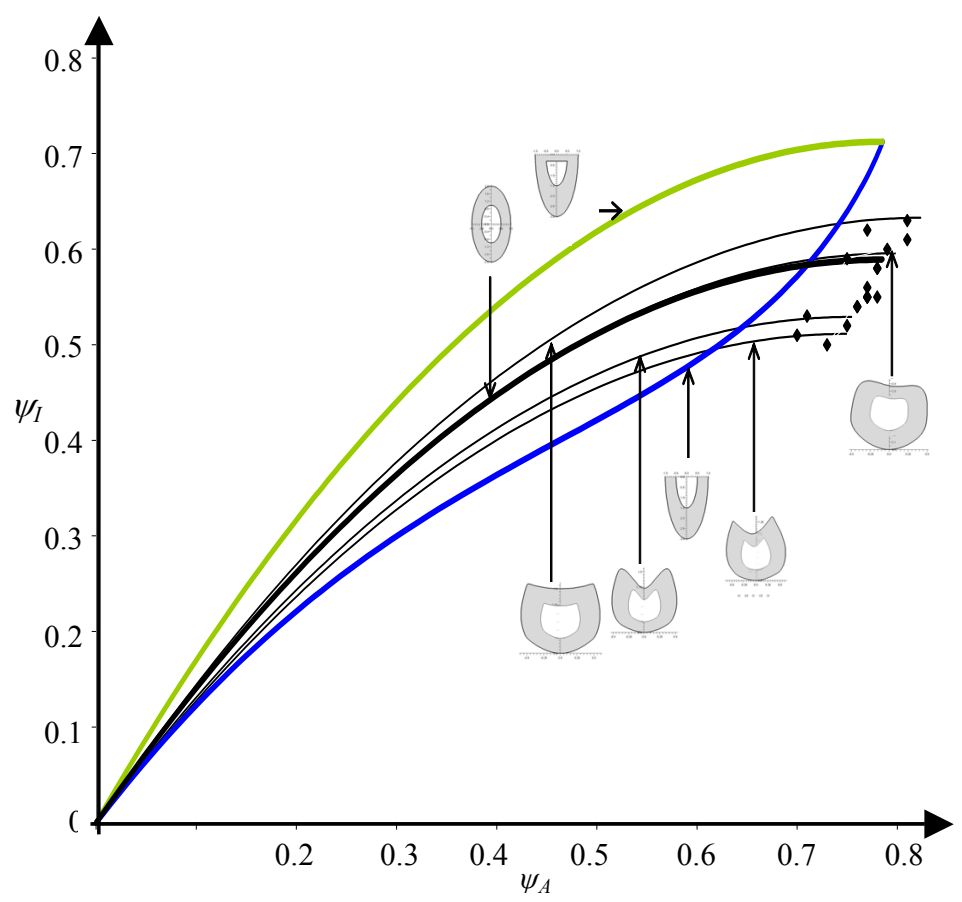

Figure 3: $\quad$ Flexural stiffness of petioles and specimen (Tables 1-2). Isotropic material.

\subsection{Structured layered material}

The effect of structuring and layering elliptical and semielliptical shapes is illustrated in Figures 5 for the flexural stiffness. If the solid cross-sections, A and $\mathrm{C}$, are structured with a network of solid circular cells, then the properties of the homogenous material are transformed into those of the structured materials $B_{0}$ and $\mathrm{D}_{0}$ respectively. The paths followed from $\mathrm{A}$ to $\mathrm{B}$ and from $\mathrm{C}$ to $\mathrm{D}$ demonstrate that layering is governed by the relative change of the outer coating thickness with respect to thickness of the inner layer. If rather than solid, hollow cells are considered, then for a given envelope and amount of material, structuring increases the flexural stiffness of a petiole. Maximizing $d(d \rightarrow 1)$, i.e. minimizing the cell walls, results in a left-oriented shift of B and D toward the origin. For example, if the relative cell thickness $d=h / H$ changes discretely from 0.5 to 0.8 , then structuring $\mathrm{A}$ and $\mathrm{C}$ has the effect of improving the flexural stiffness to $\mathrm{B}_{1}, \mathrm{~B}_{2}$, and $\mathrm{D}_{1}, \mathrm{D}_{2}$.

Figure 6 shows that structuring has a greater impact on the flexural stiffness than on the torsional stiffness. For structured and homogenous material crosssections of hollow elliptical shapes no impact on the twist to bend ratio is observed regardless of size. On the other hand, for the asymmetric shapes in Figure 6, flattening increases the torsional compliance. Hence, the twist to bend 


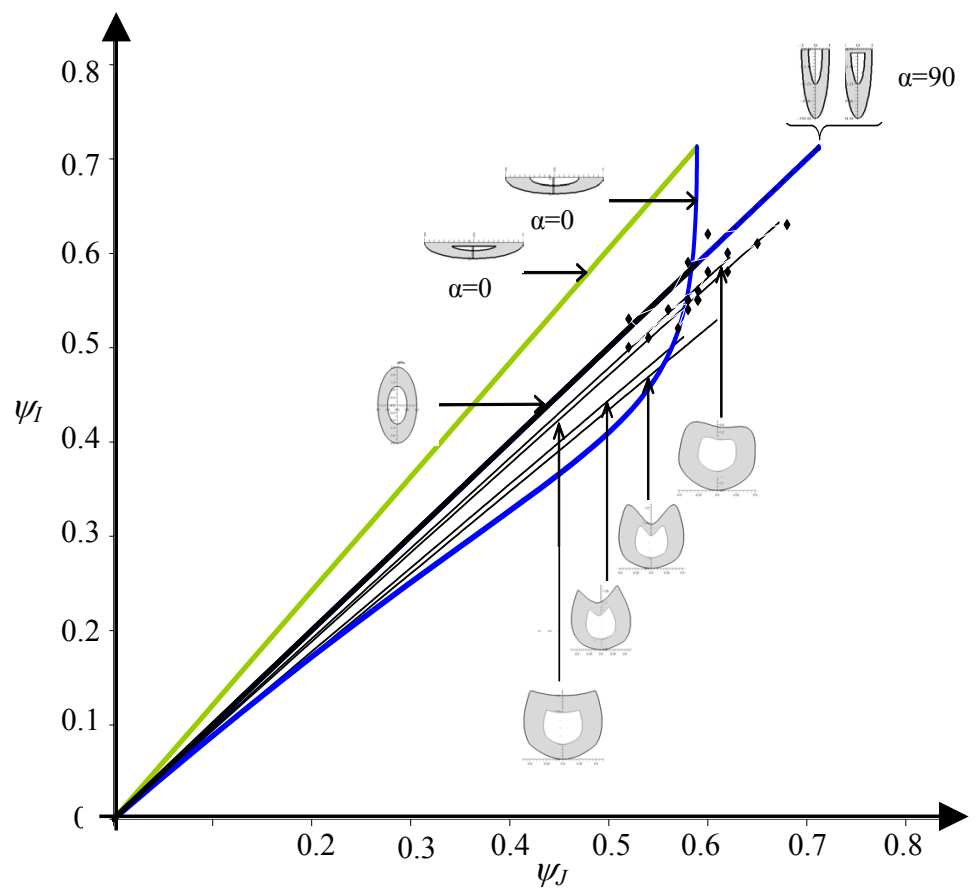

Figure 4: Flexural vs. torsional stiffness of petioles and specimen (Tables 1-2).

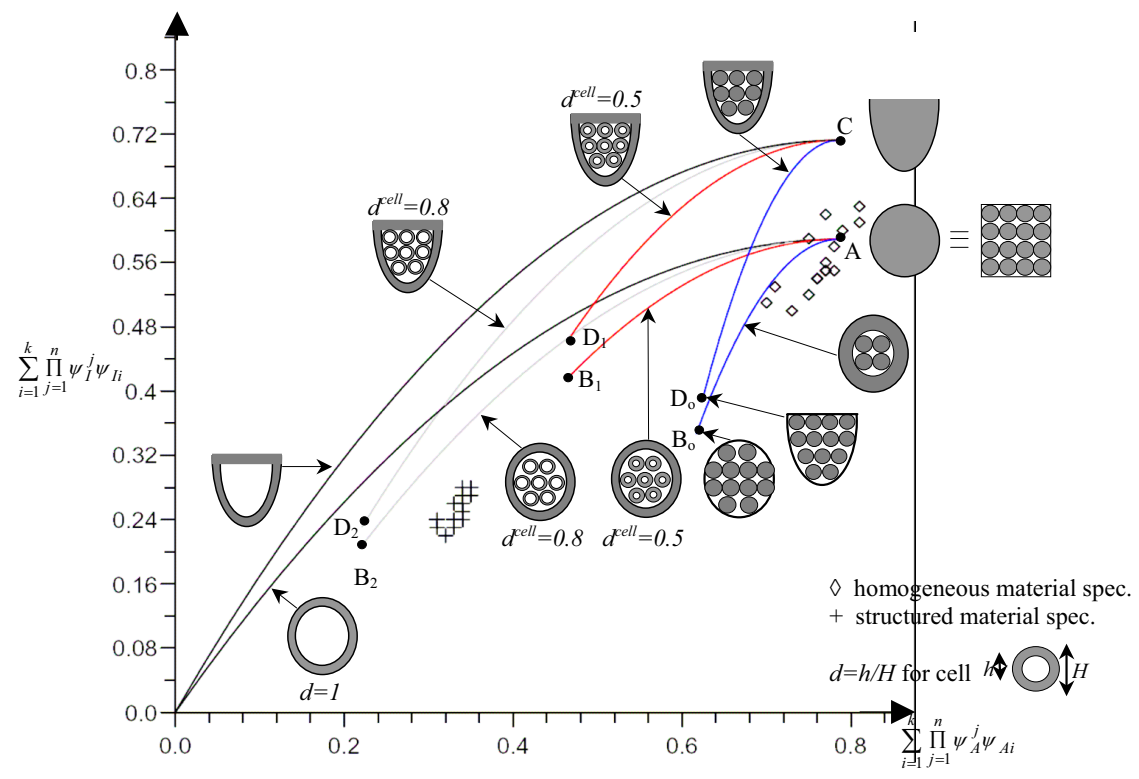

Figure 5: $\quad$ Flexural stiffness of structured petioles and specimen (Tables 1-2).

WIT Transactions on Ecology and the Environment, Vol 114, (c) 2008 WIT Press www.witpress.com, ISSN 1743-3541 (on-line) 


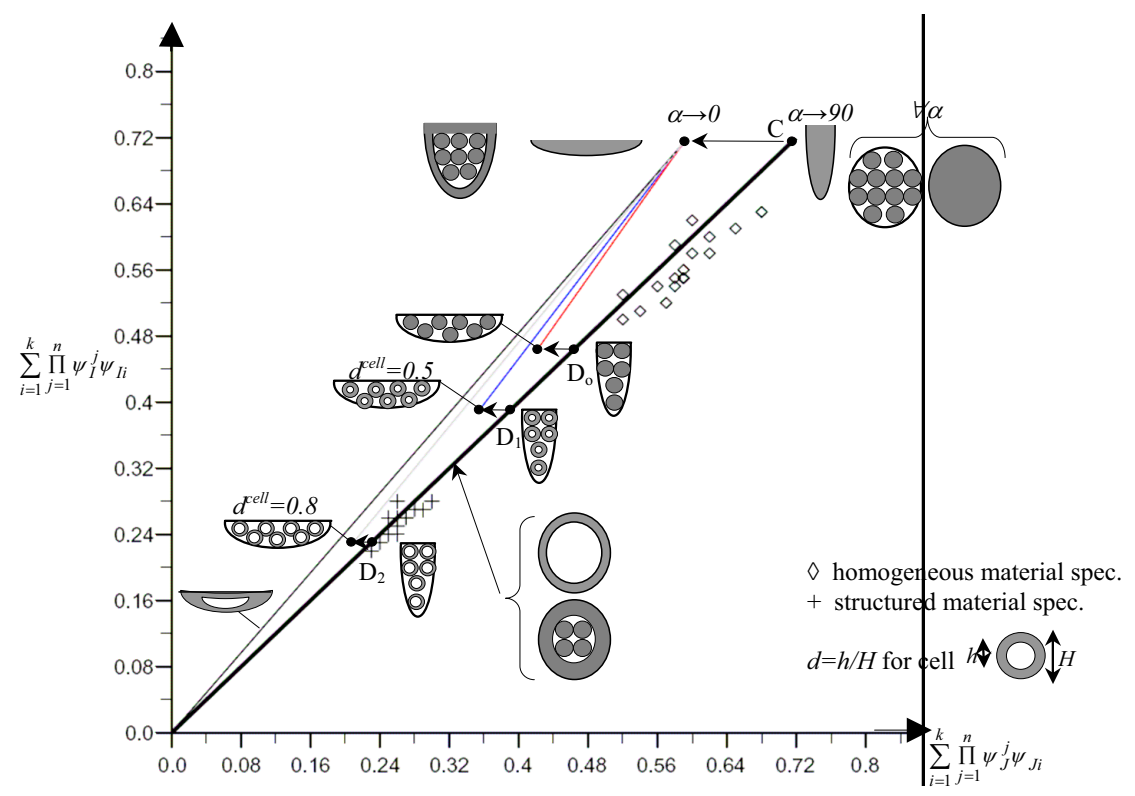

Figure 6: $\quad$ Flexural stiffness of structured petioles and specimen (Tables 1-2).

ratio rises for a combined effect of increasing flexural stiffness by structuring and decreasing the torsional rigidity by shape flattening and grooving. Table 1 gives specimen data for uniform and structured not layered materials with hollow cell walls of $\mathrm{d}=0.8$. They are identified in Figures 5 and 6 with diamonds and crosses.

A careful examination of the chart provides useful insights, which can help reveal the efficient design strategies employed by Nature. Understanding how Nature lowers $\mathrm{G}$ while betters $\mathrm{E}$ is crucial to the synthesis of novel biomimetic structures.

\section{Concluding remarks}

Like many natural materials, the petiole can be considered as a hierarchical structure. In this paper, we modelled different hierarchical levels. At the microscopic level, the material has been assumed as a cellular solid, where the basic component, cellulose, is structured into clusters of hollow tubes. At the level of the cross-section, the petiole geometry is parameterized by using the Gielis formulation, which can describe natural forms of grooved petioles for various species with high accuracy. The proposed multiscale model, which characterizes the physical properties of hierarchical layered structures, has been applied to examine the compliance properties of petioles from different species. Future work will address the impact of nano-scale structural motifs, such as the spiral reinforcements of cells, on the twist to bend ratio. 


\section{Acknowledgements}

The author thanks Mr. Arman Oduncuoglu and Mr. Vikram Chopra for their help in finalizing tables and figures.

\section{References}

[1] Vogel, S., 2007, J. Biosci., 2007, 32 (4), 634-655.

[2] Vogel, S. 1984, Am. Zool. 24: 37-44.

[3] Ennos, A.R., 1993, Annals of Botany, 72(2), 123-127.

[4] Etnier, S. A., 2001, Biological Bulletin, 200(1), 1-8.

[5] S. A. Etnier, 2003, Biol. Bull., 205(1), 36-46.

[6] Vogel, S., 1995 J. of Experimental Botany, 46

[7] Windsor-Collins A.G., Atherton M.A., Collins M.W., Cutler D.F, (2007), Int. Journal of Design and Nature, Vol. 2, Issue 4, 328-347.

[8] Pasini D., 2008, Int. Journal of Design and Nature and Ecodynamics, vol 1, in press.

[9] Aizenberg J, Weaver J. C., Thanawala M. S., Sundar V. C., Morse D. E., Fratz P., 2005, Science, Vol. 309. no. 5732, 275-278.

[10] Parkhouse, J.G, 1984, Proc. 3rd Int. Conf. On Space Structures, H. Nooshin. Elsevier Applied Science Publishers, 367374

[11] Lakes, R., 1993, Nature. Vol. 361, 511-515

[12] Gibson, L. J. and Ashby, M. F., 1997, Cellular Solids: Structure and Properties, Cambr. Un. Pr.

[13] Pasini D., 2007, Journal of Materials and Design, Vol 28, Issue 7, 20712079.

[14] Pasini D., 2006, Journal of Microelectromechanical Systems, 15 (6), pp. $1745-1758$.

[15] Gielis, J., 2003, Amer. J. Botany, 90, 333-338. 